\title{
The effect of nonsurgical periodontal treatment on serum and saliva chitotriosidase activities in patients with periodontitis and coronary artery disease
}

\author{
Mehmet Vehbi Bal' \\ Abdullah Olgun² \\ Dilek Abaslı ${ }^{3}$ \\ Atilla Özdemir ${ }^{1}$ \\ Hürkan Kürșaklıoğlu ${ }^{4, \dagger}$ \\ Ahmet Salim Göktepe ${ }^{5}$ \\ Ísmail Kurt ${ }^{3}$ \\ 'Department of Periodontology, \\ ${ }^{2}$ Biochemistry Laboratory, TSK \\ Rehabilitation Center, ${ }^{3}$ Department \\ of Biochemistry and Clinical \\ Biochemistry, ${ }^{4}$ Department of \\ Cardiology, ${ }^{5}$ Department of \\ Orthopedics, TSK Rehabilitation \\ Center, Gülhane Military Medical \\ Academy, Ankara, Turkey
}

†Hürkan Kürșaklıoğlu passed away on July I7, 2012
This article was published in the following Dove Press journal:

Therapeutics and Clinical Risk Management

30 December 2014

Number of times this article has been viewed

Background: Plasma chitotriosidase activity, which is a marker of macrophage activation, has been reported to increase in inflammatory conditions and atherosclerosis. Chronic periodontitis has likely an important role in the development of coronary artery disease. In this study, we aimed to analyze the effect of chronic periodontitis on salivary and plasma chitotriosidase activities in patients with or without coronary atherosclerosis.

Methods: Fifty subjects were divided into four groups as controls $(n=13)$, periodontitis $(n=11)$, coronary artery disease $(n=13)$, and periodontitis + coronary artery disease $(n=13)$. Plasma and saliva chitotriosidase activities were measured by a fluorimetric method in all groups before the nonsurgical treatment of periodontitis and 5 weeks posttreatment in periodontitis groups.

Results: Salivary chitotriosidase activity was decreased after nonsurgical periodontal treatment in patients having periodontitis with or without coronary atherosclerosis. However, plasma activities remained unchanged.

Conclusion: Although this study has some limitations like small sample size and short study duration, it can suggest that salivary chitotriosidase can have the potential to be used as a very useful and practical marker to evaluate the success of the periodontal treatment and/or host response.

Key finding: Salivary chitotriosidase can be used as a marker for the evaluation of the success of the periodontal treatment and/or host response.

Keywords: chitotriosidase, chronic periodontitis, coronary artery disease, nonsurgical periodontal treatment

\section{Introduction}

Chitinases hydrolyze chitin that is a structural polymer of $N$-acetyl glucosamine. Different lower organisms synthesize chitin. In mammals, although there is no endogenous chitin, there are two active chitinases. The first discovered mammalian chitinase is plasma chitotriosidase and is found to be very significantly increased in Gaucher disease that is a lysosomal storage disease. The second chitinase in mammals is acidic mammalian chitinase that is active at acidic $\mathrm{pH}$ and expressed in gastrointestinal tract and the lung. The only chitinase in human blood is chitotriosidase. Although it was reported to possess antifungal activity, $>5 \%$ of most ethnic groups lack it. ${ }^{1-4}$

Periodontal disease was reported to have a possible role in the pathogenesis of atherosclerosis, ${ }^{5-7}$ and chitotriosidase was shown to increase in both periodontitis ${ }^{8,9}$ and atherosclerosis. ${ }^{10,11}$ However, the relation between periodontitis treatment, atherosclerosis, and plasma or salivary chitotriosidase was not studied extensively.
Correspondence: Abdullah Olgun Biogerontology Laboratory, ARGE-I Building, No 2/I, Technocity, Kampus, Akdeniz University, Antalya, Turkey

Tel +90505 6695248

$\mathrm{Fax}+90242310|56|$

Email aolgun@yahoo.com 
Therefore, in this study, we aimed to investigate the effect of nonsurgical periodontal treatment on salivary and plasma chitotriosidase activities in periodontitis patients with or without clinically diagnosed coronary artery disease (CAD) (Figure 1).

\section{Materials and methods Subjects}

This was a prospective controlled clinical study. The study was approved by the Ethical Committee of Gülhane Military Medical Academy and performed according to the principles of the latest version of the Declaration of Helsinki. The subjects of the study were selected from volunteers who routinely attended the outpatient clinic of the Department of Periodontology of Gülhane Military Medical Academy. According to diagnosis, the participants were divided into four groups as follows: periodontitis $(\mathrm{P}, \mathrm{n}=11$, six males, five females): individuals are systemically healthy, $\geq 5 \mathrm{~mm}$ periodontal pockets on at least two teeth in every half jaw; periodontitis + coronary artery disease $(\mathrm{P}+\mathrm{CAD}, \mathrm{n}=13$, all males): individuals with no systemic health problems except $\mathrm{CAD}, \geq 5 \mathrm{~mm}$ periodontal pockets on at least two teeth in every half jaw; coronary artery disease $(\mathrm{CAD}$, $\mathrm{n}=13$, ten males, three females): individuals diagnosed with CAD and considered healthy after clinical and radiological examinations in periodontology clinic; and otherwise healthy controls $(C, n=13$, ten males, three females): individuals are systemically and periodontally healthy. CAD was diagnosed by angiography (having $>50 \%$ obstruction in at least one coronary artery) in the Department of Cardiology. To ensure that the individuals with $\mathrm{CAD}$ are maintaining their health, drugs of vital importance were not suspended. Informed consents of all participants were obtained. It was observed that all individuals included in whole study:

- have not been using antibiotics and anti-inflammatory drugs within the last 6 months,

- have not underwent periodontal treatment within the last 6 months, and

- are not pregnant, breastfeeding, and are not using oral contraceptives.

\section{Assessment of periodontal condition}

Periodontal indexes and clinical measurement values of individuals voluntarily participating in the research were recorded to patient follow-up form before and after treatment. Periodontal conditions were assessed using Williams

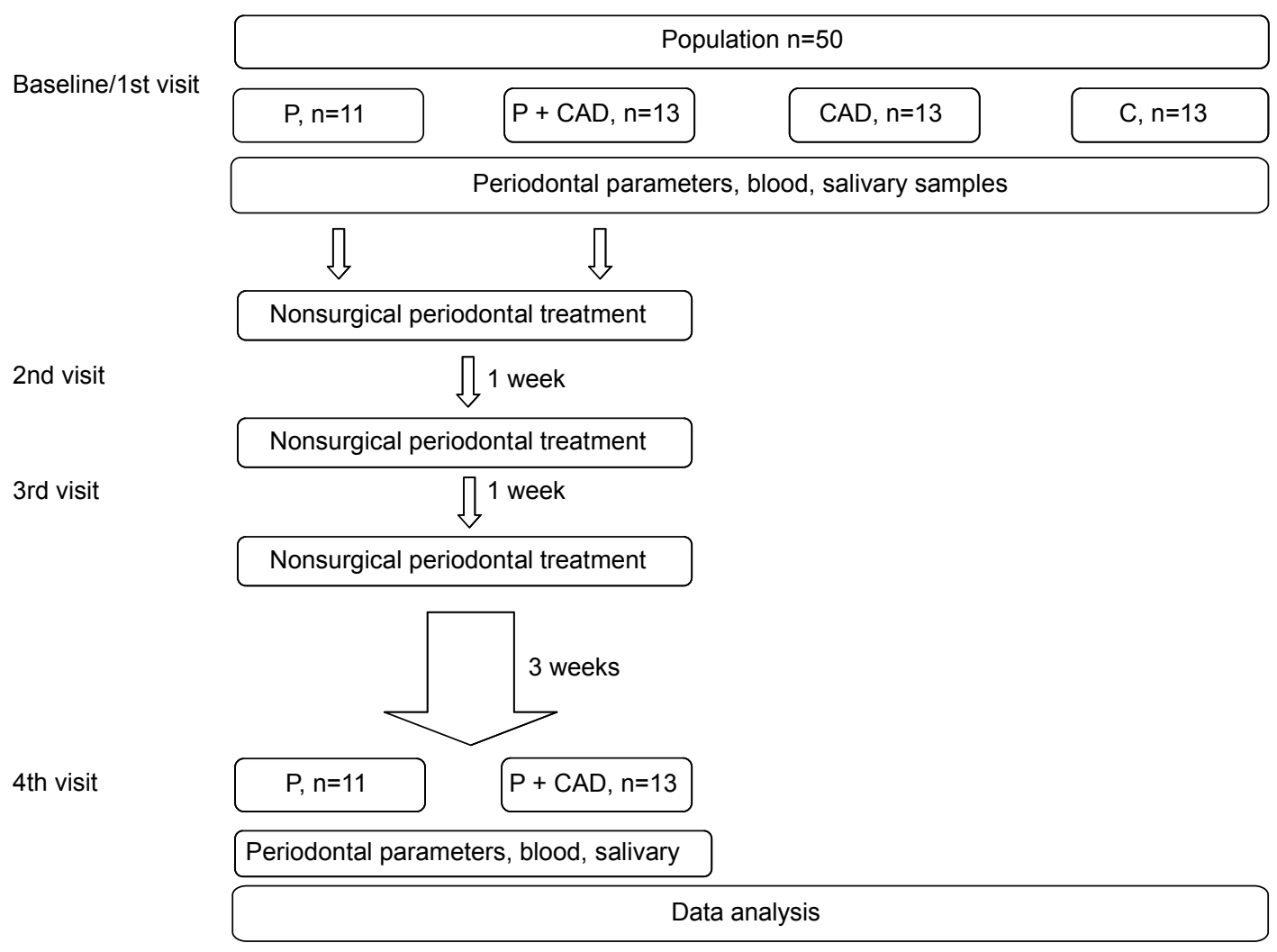

Figure I Study design: study flow diagram and study timeline.

Abbreviations: $\mathrm{P}$, periodontitis; CAD, coronary artery disease; C, controls. 
periodontal probe with a diameter of $0.5 \mathrm{~mm}$ (Hu-Friedy Ins Co, USA) on four points of every teeth, mesio-buccal, buccal, disto-buccal, and lingual or palatinal, except third molar teeth. Plaque index, ${ }^{12}$ gingival index, ${ }^{13}$ and probing depth were measured in order to determine periodontal health. Patients with periodontitis were treated nonsurgically. Posttreatment analyses were performed after 5 weeks from the beginning of treatments.

\section{Periodontal treatment procedure applied for individuals with periodontal disease}

After evaluating radiographic examinations and periodontal conditions and taking saliva and blood samples from individuals with periodontal disease, oral hygiene training, which is the first step of nonsurgical periodontal treatment, was provided. As a method of brushing teeth, the individuals were trained about the use of modified Bass technique, ${ }^{14}$ dental floss, and/or interdental brush through a model.

After oral hygiene training, scaling and root planning (SRP) was performed by using root planning after anesthesia on pockets with a dimension of $\geq 5 \mathrm{~mm}$ (Gracey, 1/2, 3/4, 5/6, 7/8, 9/10, 11/12, 13/14; Hu-Friedy Ins Co) with ultrasonic device (EMS SA CH, Nyon, Switzerland). After SRP, each periodontal pocket was cut from the center by $2 \mathrm{~cm}^{3}$ sterile tip dental injector diamond burr. With an angle of $135^{\circ}$, $1 \mathrm{~mL}$ of $0.2 \%$ chlorhexidine (Klorhex; Drogsan, Turkey) was withdrawn $1 \mathrm{~mm}$ from the base of pocket during 20 seconds, and irrigation was performed once a week. ${ }^{15}$ In addition, early contacts, which are significant in pretreatment and may result in occlusal trauma, were removed, and restorations, which may lead to plaque accumulation, were corrected. $\mathrm{P}$ and $\mathrm{P}+\mathrm{CAD}$ groups were called for additional sessions, and they were asked to bring recommended toothbrush, dental floss, and interdental brush. In examinations of individuals, it was evaluated if removal of the microbial dental plaque without staining was effective. Areas, where deficiency and inadequacy were determined, were shown to individuals, and effective removal of microbial dental plaque and its significance were stated again. Individuals, for whom preliminary periodontal treatment was applied, were not given any antibiotics in addition to the treatment.

Medical history information obtained from patients in the beginning was recorded in patient follow-up form. Panoramic films were taken from patients. Plasma and saliva samples were taken from patients. Clinical indexes and measurements were performed, and nonsurgical periodontal treatment was applied. After a week, they were called for second session, and nonsurgical periodontal treatment was repeated. After a week, they were called for third session, checks were performed, nonsurgical periodontal treatment was repeated for the patients required, and they were asked to wait for a recovery period of 3 weeks. Panoramic films and plasma and saliva samples were taken from the patients who were called for fourth session, and clinical indexes and measurements were repeated. Periodontal treatment was not applied for CAD and $\mathrm{C}$ group patients. Medical history information obtained from patients was recorded in patient follow-up form. Panoramic films were taken from patients. Plasma and saliva samples were taken from patients. Clinical indexes and measurements were performed (Figure 1).

\section{Measurements}

Blood was withdrawn into $\mathrm{K}_{3}$ EDTA-anticoagulated, evacuated blood collection tubes after an overnight fasting; plasma samples were separated immediately by centrifugation at $4^{\circ} \mathrm{C}$ and 4,000 rpm for 6 minutes and stored frozen at $-25^{\circ} \mathrm{C}$ until analysis. Saliva samples were taken after an overnight fasting and 5 minutes after a mouthwash with distilled water, and stored directly as plasma samples. Chitotriosidase activities in plasma and saliva were measured by a fluorimetric method, as previously described. Briefly, $5 \mu \mathrm{L}$ of plasma or saliva were incubated with $100 \mu \mathrm{L}$ of $22 \mu \mathrm{mol} / \mathrm{L} 4$-methylumbelliferyl$\beta$-D- $N, N^{\prime}, N^{\prime \prime}$-triacetylchitotriose (Sigma M-5639) in McIlvain's phosphate-citrate buffer, $\mathrm{pH} 5.2$, for 1 hour at $37^{\circ} \mathrm{C}$. Reactions were terminated by adding $120 \mu \mathrm{L}$ of $0.5 \mathrm{~mol} / \mathrm{L} \mathrm{Na}_{2} \mathrm{CO}_{3}-\mathrm{NaHCO}_{3}$ buffer, $\mathrm{pH} 10.7$, and the fluorescence of 4-methylumbelliferon (MU) was measured in a Microfluor $2^{\circledR}$ fluorimeter (Bio-Tek Synergy ${ }^{\mathrm{HT}}$; excitation $355 \mathrm{~nm}$, emission $460 \mathrm{~nm}$ ). The chitotriosidase activity was expressed as $\mathrm{mU} / \mathrm{mL}$ and $\mathrm{U} / \mathrm{g}$ protein. One unit of enzyme activity corresponded to the liberation of $1 \mu \mathrm{mol} \mathrm{MU} / \mathrm{min}$. Protein was determined according to Lowry et al. ${ }^{16}$

\section{Statistical analysis}

The differences between pre- and posttreatment values of $\mathrm{P}$ and $\mathrm{P}+\mathrm{CAD}$ groups were tested for significance by Wilcoxon signed ranks test. The differences among all four groups were tested for significance by Kruskal-Wallis and Bonferroni-adjusted Mann-Whitney $U$-test. The comparisons of posttreatment values of $\mathrm{P}$ and $\mathrm{P}+\mathrm{CAD}$ groups with $\mathrm{CAD}$ and $\mathrm{C}$ groups were done separately in order to check if periodontal treatment decreased the values to the levels of control groups. Statistical analysis was performed by using the SPSS 13.0 Statistical Package Program for Windows (SPSS Inc., Chicago, IL, USA). The statistical level of significance was defined as $P<0.05$. 
Table I The plasma chitotriosidase before and after nonsurgical periodontitis treatment in study groups

\begin{tabular}{|c|c|c|c|c|c|}
\hline & $\mathbf{P}, \mathrm{n}=\mathbf{I} \mathbf{I}$ & $P+C A D, n=13$ & CAD, $n=13$ & $C, n=13$ & $P$-value ${ }^{a}$ \\
\hline \multicolumn{6}{|c|}{ Plasma chitotriosidase $(\mathrm{mU} / \mathrm{mL})$} \\
\hline Before treatment & $2.9 \pm 1.7$ & $2.9 \pm 1.9$ & $2.6 \pm 2.3$ & $3.8 \pm 4.3$ & 0.873 \\
\hline After treatment & $3.4 \pm 2.0$ & $2.7 \pm 1.5$ & & & 0.713 \\
\hline$P$-value ${ }^{b}$ & 0.461 & 0.593 & & & \\
\hline
\end{tabular}

Notes: ${ }^{\mathrm{a}} \alpha: 0.05 / 6=0.008$ after Bonferroni adjustment in pairwise comparisons. The comparisons of posttreatment values of $P$ and $P+C A D$ groups were done with pretreatment values of CAD and $C$ groups. ${ }^{b}$ Comparison of pre- and posttreatment values in the same group.

Abbreviations: $\mathrm{P}$, periodontitis; $\mathrm{CAD}$, coronary artery disease; C, controls.

\section{Results}

The mean age of the study groups was similar, except significantly higher mean age of $\mathrm{P}+\mathrm{CAD}$ group compared with control group $(P=0.001)$. The mean ages of $\mathrm{P}, \mathrm{P}+\mathrm{CAD}$, $\mathrm{CAD}$, and $\mathrm{C}$ groups were $47.9 \pm 9.3$ years, $58.0 \pm 7.6$ years, $56.2 \pm 9.9$ years, and $44.4 \pm 8.7$ years, respectively.

\section{Plasma chitotriosidase activity}

The plasma chitotriosidase activities were not different among all groups and in $\mathrm{P}$ and $\mathrm{P}+\mathrm{CAD}$ groups before and after nonsurgical periodontal treatment (Table 1).

\section{Salivary chitotriosidase activity}

The salivary chitotriosidase activities, however, were significantly higher in periodontal disease group than control group before periodontal treatment $(P=0.001$ and $P=0.003$ in both as $\mathrm{mU} / \mathrm{mL}$ concentration and corrected per protein $[\mathrm{U} / \mathrm{g}]$, respectively, for $\mathrm{P}$ and $\mathrm{C}$ ) (Table 2). Periodontal treatment significantly decreased salivary chitotriosidase activities in both $\mathrm{P}$ (although not significant when activity was represented as $\mathrm{mU} / \mathrm{mL}, P=0.056$; becomes significant after correction as per protein $[\mathrm{U} / \mathrm{g}], P=0.021)$ and $\mathrm{P}+\mathrm{CAD}$ groups ( $P=0.005$ in both as $\mathrm{mU} / \mathrm{mL}$ concentration and corrected per protein $[\mathrm{U} / \mathrm{g}])$. But periodontal treatment failed to decrease salivary chitotriosidase activities to the levels of the nonperiodontal disease groups $(P=0.049$ and $P=0.015$ in both as $\mathrm{mU} / \mathrm{mL}$ concentration and corrected per protein $[\mathrm{U} / \mathrm{g}]$, respectively, for $\mathrm{P}$ and $\mathrm{CAD}$; and similarly, $P=0.002$ and $P=0.030$, respectively, for $\mathrm{P}$ and $\mathrm{C}$ ) (nonsignificant $P$-values are not shown in Table 2). Therefore, salivary chitotriosidase activities after treatment were still significantly higher than control groups.

Salivary protein concentrations were not different among all groups before treatment and in $\mathrm{P}$ and $\mathrm{P}+\mathrm{CAD}$ groups before and after nonsurgical periodontal treatment (Table 2).

\section{Periodontal indexes}

The periodontal indexes, which are plaque index, gingival index, and probing depth, generally showed that periodontal health was deteriorated mostly in $\mathrm{P}$ and $\mathrm{P}+\mathrm{CAD}$ groups as expected (Table 3). According to these indexes, periodontal health was also significantly worse than control group. All of these periodontal indexes that were measured to evaluate the success of periodontal treatment were significantly improved after treatment in both $\mathrm{P}$ and $\mathrm{P}+\mathrm{CAD}$ groups $(P \leq 0.005$ for all indexes) (Table 3).

Table 2 The salivary chitotriosidase and salivary protein before and after nonsurgical periodontitis treatment in study groups

\begin{tabular}{|c|c|c|c|c|c|}
\hline & $\mathbf{P}, \mathbf{n}=\mathbf{I} \mathbf{I}$ & $P+C A D, n=13$ & CAD, $n=13$ & C, $n=13$ & $P$-value \\
\hline \multicolumn{6}{|c|}{ Salivary chitotriosidase $(\mathrm{mU} / \mathrm{mL})$} \\
\hline Before treatment & $8.2 \pm 6.2$ & $4.6 \pm 4.2$ & $3.8 \pm 6.5$ & $1.4 \pm 2.2$ & $0.005^{\mathrm{b}}$ \\
\hline After treatment & $5.8 \pm 4.2$ & $1.4 \pm 1.8$ & & & $0.014^{c}$ \\
\hline$P$-value ${ }^{d}$ & 0.056 & 0.005 & & & \\
\hline \multicolumn{6}{|c|}{ Salivary chitotriosidase per protein $(\mathrm{U} / \mathrm{g})$} \\
\hline Before treatment & $5.3 \pm 3.0$ & $3.1 \pm 2.6$ & $1.5 \pm 2.0$ & $2.0 \pm 1.8$ & $0.004^{e}$ \\
\hline After treatment & $3.9 \pm 2.4$ & $1.4 \pm 1.3$ & & & $0.018^{f}$ \\
\hline$P$-value ${ }^{d}$ & 0.021 & 0.005 & & & \\
\hline \multicolumn{6}{|c|}{ Salivary protein (mg/mL) } \\
\hline Before treatment & $1.5 \pm 0.6$ & $1.4 \pm 0.5$ & $1.3 \pm 0.8$ & $1.0 \pm 0.3$ & 0.104 \\
\hline After treatment & $1.4 \pm 0.5$ & $1.0 \pm 0.4$ & & & 0.127 \\
\hline$P$-value ${ }^{b}$ & 0.594 & 0.308 & & & \\
\hline
\end{tabular}

Notes: ${ }^{\mathrm{a}} \alpha: 0.05 / 6=0.008$ after Bonferroni adjustment in pairwise comparisons. The comparisons of posttreatment values of $\mathrm{P}$ and $\mathrm{P}+\mathrm{CAD}$ groups were done with pretreatment values of $C A D$ and $C$ groups. ${ }^{b} P=0.001$ for $P$ vs $C$. ${ }^{P} P=0.005$ for $P$ vs $P+C A D$ and $P=0.002$ for $P$ vs $C$. ${ }^{d}$ Comparison of pre- and posttreatment values in the same group. ${ }^{e}=0.002$ for $P$ vs $C A D$ and $P=0.003$ for $P$ vs $C$. $P=0.005$ for $P$ vs $P+C A D$.

Abbreviations: $P$, periodontitis; $C A D$, coronary artery disease; $C$, controls. 
Table 3 The periodontal indexes before and after nonsurgical periodontitis treatment in study groups

\begin{tabular}{|c|c|c|c|c|c|}
\hline & $\mathbf{P}, \mathbf{n}=\mathbf{I} \mathbf{I}$ & $P+C A D, n=13$ & CAD, $n=13$ & $C, n=13$ & $P$-value ${ }^{a}$ \\
\hline \multicolumn{6}{|l|}{ Plaque index } \\
\hline Before treatment & $2.1 \pm 0.4$ & $1.7 \pm 0.6$ & $1.1 \pm 0.4$ & $0.3 \pm 0.2$ & $<0.001^{\mathrm{b}}$ \\
\hline After treatment & $0.4 \pm 0.6$ & $0.5 \pm 0.4$ & & & $<0.001^{c}$ \\
\hline$P$-value ${ }^{d}$ & 0.005 & 0.002 & & & \\
\hline \multicolumn{6}{|l|}{ Gingival index } \\
\hline Before treatment & $1.9 \pm 0.7$ & $1.5 \pm 0.6$ & $0.8 \pm 0.4$ & $0.3 \pm 0.2$ & $<0.001^{\mathrm{e}}$ \\
\hline After treatment & $0.4 \pm 0.2$ & $0.4 \pm 0.3$ & & & $<0.00 \mathrm{I}^{\mathrm{f}}$ \\
\hline$P$-value ${ }^{b}$ & 0.003 & 0.002 & & & \\
\hline \multicolumn{6}{|l|}{ Probing depth } \\
\hline Before treatment & $3.5 \pm 0.6$ & $3.2 \pm 0.9$ & $1.5 \pm 0.3$ & $1.1 \pm 0.2$ & $<0.001^{g}$ \\
\hline After treatment & $2.0 \pm 0.7$ & $1.8 \pm 0.4$ & & & $<0.00 \mathrm{I}^{\mathrm{h}}$ \\
\hline$P$-value ${ }^{b}$ & 0.003 & 0.002 & & & \\
\hline
\end{tabular}

Notes: ${ }^{\mathrm{a}} \alpha: 0.05 / 6=0.008$ after Bonferroni adjustment in pairwise comparisons. The comparisons of posttreatment values of $P$ and $P+C A D$ groups were done with pretreatment values of $C A D$ and $C$ groups. ${ }^{b} P<0.00 I$ for $P$ vs $C A D, P$ vs $C, P+C A D$ vs $C$, and $C A D$ vs $C$. ${ }^{c} P=0.00$ I for $P$ vs $C A D, P=0.007$ for $P+C A D$ vs $C A D$, and $P<0.00 I$ for $C A D$ vs $C$. ${ }^{d}$ Comparison of pre- and posttreatment values in the same group. ${ }^{e}<0.00$ I for $P$ vs $C A D, P$ vs $C, P+C A D$ vs $C$, and $C A D$ vs $C ; P=0.006$ for $P+C A D$ vs $C A D$. ${ }^{f} P=0.01$ I for $P$ vs $C A D, P=0.003$ for $P+C A D$ vs $C A D$, and $P<0.001$ for $C A D$ vs $C$. ${ }^{8} P<0.00$ I for $P$ vs $C A D, P$ vs $C, P+C A D$ vs $C A D$, and $P+C A D$ vs $C$; $P=0.002$ for $C A D$ vs $C$. ${ }^{h} P<0.001$ for $P$ vs $C$ and $P+C A D$ vs $C ; P=0.002$ for $C A D$ vs $C$.

Abbreviations: $P$, periodontitis; $C A D$, coronary artery disease; $C$, controls.

\section{Discussion}

In this prospective controlled study, we evaluated the change in salivary and plasma chitotriosidase activities with nonsurgical periodontal treatment in patients with periodontitis. Since periodontal disease is related to atherosclerosis, and chitotriosidase increases in both periodontitis and atherosclerosis, we determined salivary and plasma chitotriosidase activities in periodontitis patients with or without clinically diagnosed CAD. In this way, we aimed to discriminate the effect of CAD on chitotriosidase activities. We found that plasma chitotriosidase activities were not different among all groups and in $\mathrm{P}$ and $\mathrm{P}+\mathrm{CAD}$ groups before and after nonsurgical periodontal treatment. This clearly shows that periodontitis does not have an effect on plasma chitotriosidase activity.

The structure of human chitotriosidase has been characterized in detail; however, its function has not been fully defined. The chitotriosidase was hypothetized to play a role in defense against chitinous human pathogens. ${ }^{17}$ Thus, although the chitotriosidase is not metabolically essential, it may play a key role as a pathogen-defense protein and may be used as a marker of some infectious diseases and their response to treatment.

Although higher levels of plasma chitotriosidase activity were reported in atherosclerotic diseases,,${ }^{10,11}$ we could not find any difference between CAD groups and others. In our opinion, one of the reasons why baseline plasma chitotriosidase activities were not different among the groups could be the lower ratio of periodontal macrophages compared to total body macrophages that contribute to plasma chitotriosidase activities. The effect of periodontal macrophages could be negligible.

In contrary to the plasma chitotriosidase activities, our findings show that the salivary chitotriosidase activities were significantly higher in periodontal disease group than control group before periodontal treatment, and periodontal treatment significantly decreased salivary chitotriosidase activities in both $\mathrm{P}$ and $\mathrm{P}+\mathrm{CAD}$ groups. Similar results were also reported from other studies. ${ }^{89}$ Van Steijn et al $^{8}$ showed that the activity levels of chitinase in the whole saliva of periodontitis patients were significantly higher than those in saliva from controls, and periodontal treatment for a period of 5-6 months resulted in a three- to fourfold decrease in this enzyme activity. Another comparative study of whole salivas of periodontally healthy controls and gingivitis and periodontitis subjects by the same team also indicated that only in the case of periodontitis, there was a significant increase in the specific chitinase activity. ${ }^{9}$

Our findings strongly suggest that the nonsurgical treatments were likely not totally successful, and/or host response (macrophage activation) was prolonged. The normalization of host response to the levels of controls might be requiring more time. The revision of current periodontal disease treatment protocols should be considered in order to include the measures to normalize host response and stop tissue degradation, which continues even after the local eradication of pathogens. Topical use of macrophage inhibitors can be considered in addition to nonsurgical periodontal treatment.

The main limitations of the present study were small sample size of study groups and short study duration. 
Prospective studies with larger sample size and longer duration would provide further information on the change in the level of plasma chitotriosidase activity, effect of periodontitis treatment, and their relation.

As a conclusion, salivary chitotriosidase has the potential to be a relevant independent marker for the evaluation of periodontal disease treatment and destructive host response, which still continues despite the eradication of the pathogens.

\section{Disclosure}

The authors declared no conflicts of interest.

\section{References}

1. Malaguarnera L. Chitotriosidase: the yin and yang. Cell Mol Life Sci. 2006;63:3018-3029.

2. Bussink AP, Vreede J, Aerts JM, Boot RG. A single histidine residue modulates enzymatic activity in acidic mammalian chitinase. FEBS Lett. 2008;582:931-935.

3. Kurt I, Abasli D, Cihan M, et al. Chitotriosidase levels in healthy elderly subjects. Ann N Y Acad Sci. 2007;1100:185-188.

4. Guo Y, He W, Boer AM, et al. Elevated plasma chitotriosidase activity in various lysosomal storage diseases. J Inherit Metab Dis. 1995;18: 717-720.

5. Mustapha IZ, Debrey S, Oladubu M, Ugarte R. Markers of systemic bacterial exposure in periodontal disease and cardiovascular disease risk: a systematic review and meta-analysis. J Periodontol. 2007;78: 2289-2302.

6. Hettne KM, Weeber M, Laine ML, et al. Automatic mining of the literature to generate new hypotheses for the possible link between periodontitis and atherosclerosis: 1lipopolysaccharide as a case study. J Clin Periodontol. 2007;34:1016-1024.
7. Tüter G, Akan T, Kurtiş B, Toyman U, Pınar S, Okyay K. Evaluation of the oral health status in patients with coronary artery disease. Turkiye Klinikleri J Dental Sci. 2008;14:85-90.

8. Van Steijn GJ, Amerongen AV, Veerman EC, Kasanmoentalib S, Overdijk B. Effect of periodontal treatment on the activity of chitinase in whole saliva of periodontitis patients. $J$ Periodontal Res. 2002;37: 245-249.

9. Van Steijn GJ, Amerongen AV, Veerman EC, Kasanmoentalib S, Overdijk B. Chitinase in whole and glandular human salivas and in whole saliva of patients with periodontal inflammation. Eur J Oral Sci. 1999;107:328-337.

10. Karadag B, Kucur M, Isman FK, Hacibekiroglu M, Vural VA. Serum chitotriosidase activity in patients with coronary artery disease. Circ J. 2008;72:71-75.

11. Artieda M, Cenarro A, Gañán A, et al. Serum chitotriosidase activity is increased in subjects with atherosclerosis disease. Arterioscler Thromb Vasc Biol. 2003;23:1645-1652.

12. Silness J, Löe H. Periodontal disease in pregnancy. II. Correlation between oral hygiene and periodontal condition. Acta Odontol Scand. 1964;22:121-135.

13. Löe H, Silness J. Periodontal disease in pregnancy. I. Prevalance and severity. Acta Odontol Scand. 1963;21:533-551.

14. Newman MG, Takei HH, Carranza FA. Carranza's Clinical Periodontology. 9th ed. Philedelphia, PA: W.B. Saunders Company; 2002: 132-674.

15. Özdemir A, Pikdöken L, Serdar M, Akça AE. The effect of the CHX application in periodontal pockets on the gingival hydroxy proline level. J Marmara Univ Dent Fac. 2001:4:200-206.

16. Lowry OH, Rosebrouhn NJ, Farr AL, Randall RJ. Protein measurement with the Folin phenol reagent. J Biol Chem. 1951;193:265-275.

17. Fusetti F, von Moeller H, Houston D, et al. Structure of human chitotriosidase. Implications for specific inhibitor design and function of mammalian chitinase-like lectins. J Biol Chem. 2002;277:25537-25544.
Therapeutics and Clinical Risk Management

\section{Publish your work in this journal}

Therapeutics and Clinical Risk Management is an international, peerreviewed journal of clinical therapeutics and risk management, focusing on concise rapid reporting of clinical studies in all therapeutic areas, outcomes, safety, and programs for the effective, safe, and sustained use of medicines. This journal is indexed on PubMed Central, CAS,

\section{Dovepress}

EMBase, Scopus and the Elsevier Bibliographic databases. The manuscript management system is completely online and includes a very quick and fair peer-review system, which is all easy to use. Visit $\mathrm{http}: / / \mathrm{www}$.dovepress.com/testimonials.php to read real quotes from published authors. 